\title{
From animals to animats
}

Dave Cliff

Adaptive Behavior. Editor Jean-Arcady Meyer. MIT Press. 4/yr. USA \$115, Canada $\$ 138.03$, elsewhere $\$ 129$ (institutional); USA $\$ 50$, Canada $\$ 68.48$, elsewhere $\$ 64$ (personal); USA $\$ 35$, Canada $\$ 52.43$, elsewhere $\$ 49$ (student/retired).

THE scope of this journal takes some explaining. Contributions originate from a wide range of disciplines, including artificial intelligence, behavioural ecology, ethology, neuroscience, psychology and robotics. The journal's unifying theme is the study of issues in the analysis or design of autonomous agents: that is, agents

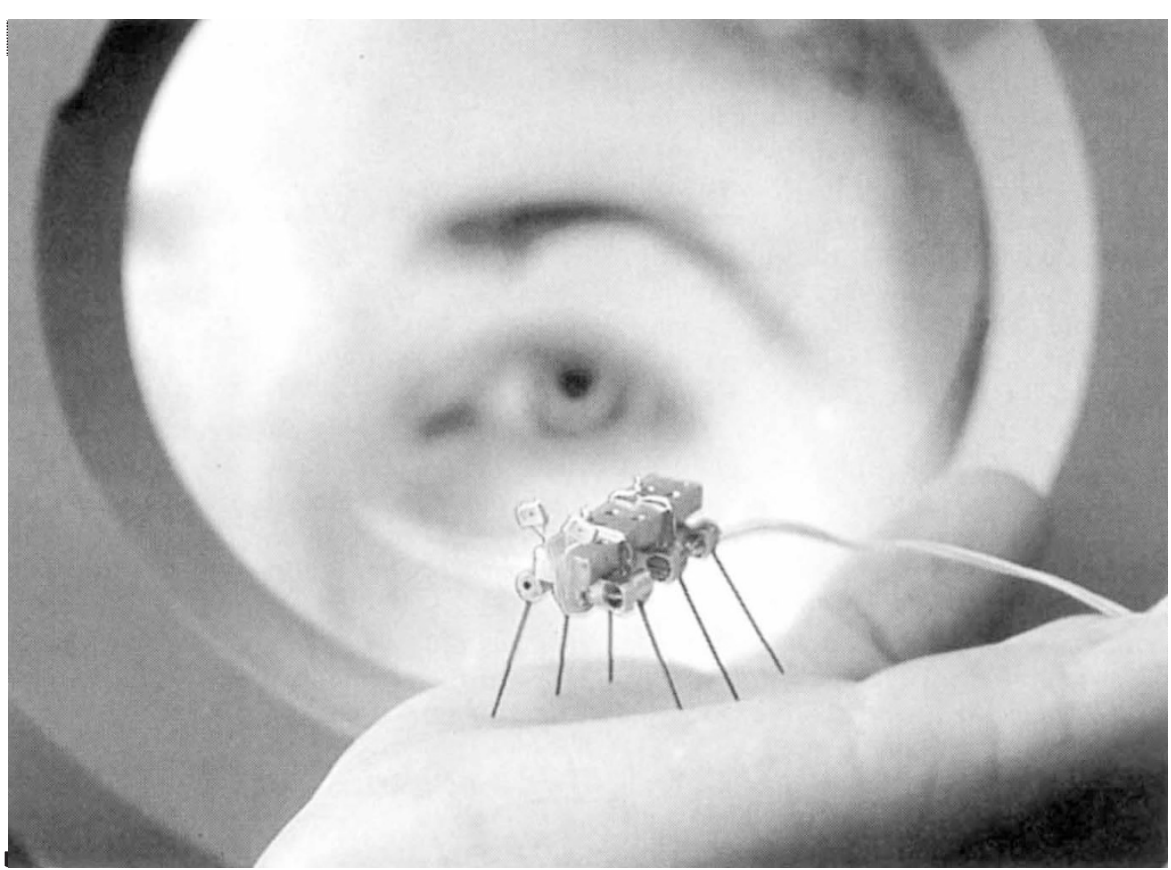

MIT-bred robot 'gnat', complete with photodetectors and logic processors.

capable of coordinating perception and action for extended periods of time in unknown and potentially hostile environments. Such coordinated activity is referred to as adaptive behaviour if it increases the agent's likelihood of survival.

The autonomous agents studied may be biological (such as animals) or artificial ("animats": creature-like mobile robots, or software agents inhabiting virtual realities). Increasingly, analysis of biological mechanisms underlying the generation of adaptive behaviours is aided by the use of computer simulations or robot models; similarly, designers of animats often draw inspiration from findings in biology. This journal offers a unique forum for the exchange of ideas and the study of adaptive behaviour in general.

Each issue in volume one contains four long articles (around 15,000 words); the first issue also contains an inaugural lead- ing article, and the last issue includes indexes to the volume. Although future volumes might see the inclusion of short papers, book reviews and so on, the decision to place early emphasis on substantial and important papers has helped to establish the journal as the authoritative publication in the field. Papers range from neurobiology to robotics, but the constant theme of understanding adaptive behaviour in both natural and artificial systems lends coherence and accessibility to research projects that might otherwise seem superficially disparate. Most of the papers published so far have been of good quality and are well written for the

जे original research papers and review articles on the effects of toxic chemicals on populations, communities and ecosystems. Neither journal is restricted to specific taxonomic groups although Journal of Aquatic Ecosystem Health is limited to aquatic systems.

Despite similar aims, the types of articles published in the first two volumes are quite different. Whereas the majority in Ecotoxicology are original research papers, most in $J A E H$ are reviews. This emphasis on review-type articles reflects the fact that seven of the first eight issues of $J A E H$ contain papers resulting from three scientific meetings. If $J A E H$ is to become more than a vehicle for conference proceedings it must begin to attract more unsolicited papers describing original research.

Both journals have a North American bias in authorship, although this is much greater for $J A E H$ ( 72 per cent compared with 44 per cent). There is also a bias in subject matter. Roughly 40 per cent of the papers in Ecotoxicology are concerned with the effects on birds of chemicals, principally pesticides. This imbalance needs to be addressed if the journal is to fulfil its intention of favouring no particular taxon or biome.

Ecotoxicology is an expanding area and there has recently been a shift from studies describing the effects of a single chemical on individual organisms to studies concerned more with understanding and predicting the effects of chemicals on populations and communities. There is therefore a need for journals that publish the results of such studies. Several established journals already do this, and unless Ecotoxicology and $J A E H$ can establish unique identities they will have difficulty competing.

There is a potential niche for publications that adopt a holistic approach to ecotoxicology, as pointed out by John Cairns $\mathrm{J} r$ in articles in the inaugural issues of these two new journals. Given their similarity in scope, however, it is unlikely 